Journal of Algebra Combinatorics Discrete Structures and Applications

\title{
Degree distance and Gutman index of two graph products
}

\section{Shaban Sedghi, Nabi Shobe}

Abstract: The degree distance was introduced by Dobrynin, Kochetova and Gutman as a weighted version of the Wiener index. In this paper, we investigate the degree distance and Gutman index of complete, and strong product graphs by using the adjacency and distance matrices of a graph.

2010 MSC: 05C07, 05C90

Keywords: Degree distance, Adjacency matrix, Distance matrix, Complete product, Strong product

\section{Introduction}

All graphs in this paper are assumed to be undirected, finite and simple. We refer to [2] for graph theoretical notation and terminology not specified here. For a graph $G$, let $V(G), E(G)$ and $\bar{G}$ denote the set of vertices, the set of edges and the complement of $G$, respectively. If $G$ is a connected graph and $u, v \in V(G)$, then the distance $d(u, v)$ between $u$ and $v$ is the length of a shortest path connecting $u$ and $v$. If $v$ is a vertex of a connected graph $G$, then the eccentricity $e(v)$ of $v$ is defined by $e(v)=\max \{d(u, v) \mid u \in$ $V(G)\}$. Furthermore, the diameter $\operatorname{diam}(G)$ of $G$ is defined by $\operatorname{diam}(G)=\max \{e(v) \mid v \in V(G)\}$.

Let $G$ be a finite, simple, connected, undirected graph with $p$ vertices and $q$ edges. In what follows, we say that $G$ is an $(p, q)$-graph. Let $V(G)=\left\{v_{1}, v_{2}, \ldots, v_{p}\right\}$ and $E(G)=\left\{e_{1}, e_{2}, \ldots, e_{q}\right\}$ be the vertex set and edge set of $G$, respectively. The adjacency matrix of $G$ is the $p \times p$ matrix $A=A(G)$ whose $(i, j)$ entry, denoted by $a_{i j}$, is defined by

$$
a_{i j}= \begin{cases}1 & \text { if } v_{i} \text { and } v_{j} \text { are adjacent } \\ 0 & \text { otherwise }\end{cases}
$$

Shaban Sedghi(Corresponding Author); Department of Mathematics, Qaemshahr Branch, Islamic Azad University, Qaemshahr, Iran (email: sedghi_gh@yahoo.com, sedghi.gh@qaemiau.ac.ir).

Nabi Shobe; Department of Mathematics, Babol Branch, Islamic Azad University, Babol, Iran (email: nabi_shobe@yahoo.com). 
The distance matrix of $G$ is the $p \times p$ matrix $D_{G}$ whose $(i, j)$ entry, denoted by $d_{i j}$, is defined by

$$
d_{i j}= \begin{cases}d_{G}\left(v_{i}, v_{j}\right) & \text { if } v_{i} \neq v_{j} \\ 0 & \text { otherwise }\end{cases}
$$

where $d_{G}\left(v_{i}, v_{j}\right)$ is the length of a shortest directed path in $G$ from $v_{i}$ to $v_{j}$.

The vertex $u$ is said to be a neighbor of $v$ if they are adjacent. The neighborhood of a vertex $v$, denoted by $N_{G}(v)$, is the set of all neighbors of $v$. The degree of a vertex $v$ in a graph $G$, denoted by $d_{v}=d_{G}(v)$, is the number of vertices in its neighborhood, that is, $d_{G}(v)=|N(v)|$. The common neighborhood graph $\operatorname{con}(G)$ (in short congraph) of a graph $G$ is defined as the graph with $V(\operatorname{con}(G))=V(G)$ and two vertices in $\operatorname{con}(G)$ are adjacent if they have a common neighbor in $G$. For every $x, y \in V(G)$,

$$
x y \in E(\operatorname{con}(G)) \text { if and only if } N_{G}(x) \cap N_{G}(y) \neq \emptyset .
$$

Some basic properties of congraphs have been established; see [1, 3].

The oldest and most studied degree-based structure descriptors are the first and second Zagreb indices [15], defined as

$$
M_{1}(G)=\sum_{v \in V(G)}\left(d_{G}(v)\right)^{2} \quad \text { and } \quad M_{2}(G)=\sum_{u v \in E(G)}\left(d_{G}(u)\right)\left(d_{G}(v)\right) .
$$

It has been shown that the first Zagreb index obeys the identity [10]

$$
M_{1}(G)=\sum_{u v \in E(G)}\left(d_{G}(u)+d_{G}(v)\right)
$$

The first investigation of the sum of distance between all pairs of vertices of a (connected) graph was done by Harold Wiener in 1947, who realized that there exists a correlation between the boiling points of paraffins and this sum [20]. Eventually, the distance-based graph invariant,

$$
W(G)=\sum_{\{u, v\} \subseteq V(G)} d(u, v) .
$$

For more details, we refer to $[8,11,13,19]$.

The degree distance was introduced by Dobrynin and Kochetova [9] and Gutman [14] as a weighted version of the Wiener index. The degree distance $D D(G)$ of a graph $G$ is defined as

$$
D D(G)=\sum_{\{u, v\} \subseteq V(G)} d_{G}(u, v)\left[d_{G}(u)+d_{G}(v)\right]=\frac{1}{2} \sum_{u, v \in V(G)} d_{G}(u, v)\left[d_{G}(u)+d_{G}(v)\right]
$$

with the summation runs over all pairs of vertices of $G$. The degree distance is also known as the Schultz index in chemical literature; see [21]. In [14], Gutman showed that if $G$ is a tree on $n$ vertices, then $D D(G)=4 W(G)-n(n-1)$; see [5,6] and [9]. In [7], Gutman index $G u t(G)$ of a graph $G$ is defined as

$$
G u t(G)=\sum_{\{u, v\} \subseteq V(G)} d_{G}(u) d_{G}(v) d(u, v) .
$$

For more details on Gutman index, we refer to [4, 7, 12].

The relations between the degree distance, Gutman index and Wiener index are shown in the following Table 1.

The join and strong products are defined as follows.

The join or complete product $G \vee H$ of two disjoint graphs $G$ and $H$, is the graph with vertex set $V(G) \cup V(H)$ and edge set $E(G) \cup E(H) \cup\{u v \mid u \in V(G), v \in V(H)\}$. 
Table 1. Three distance parameters

\begin{tabular}{|c|c|}
\hline Wiener index & $W(G)=\sum_{\{u, v\} \subseteq V(G)} d_{G}(u, v)$ \\
\hline Degree distance & $D D(G)=\sum_{\{u, v\} \subseteq V(G)} d_{G}(u, v)\left[d_{G}(u)+d_{G}(v)\right]$ \\
\hline Gutman index & $G u t(G)=\sum_{\{u, v\} \subseteq V(G)} d_{G}(u, v) d_{G}(u) d_{G}(v)$ \\
\hline
\end{tabular}

The strong product $G \otimes H$ of graphs $G$ and $H$ has the vertex set $V(G) \times V(H)$. Two vertices $(u, v)$ and $\left(u^{\prime}, v^{\prime}\right)$ are adjacent whenever $u u^{\prime} \in E(G)$ and $v=v^{\prime}$, or $u=u^{\prime}$ and $v v^{\prime} \in E(H)$, or $u u^{\prime} \in E(G)$ and $v v^{\prime} \in E(H)$.

Paulraja and Agnes [16] studied the degree distance of Cartesian and lexicographic products. Later, they [17] investigated the Gutman index of Cartesian and lexicographic products. In this paper, we investigate the degree distance and Gutman index of strong and complete product graphs.

\section{Preliminary}

We define,

$$
N_{1}(G)=\sum_{v \in V(G)} d_{G}(v) d_{\operatorname{con}(G)}(v) \quad \text { and } \quad N_{2}(G)=\sum_{u v \in E(\operatorname{con}(G))} d_{G}(u) d_{G}(v) .
$$

Definition 2.1. Let $A=\left[a_{i j}\right]_{m \times n}$. Then, we define

$$
S(A)=\sum_{1 \leq i \leq m, 1 \leq j \leq n} a_{i j}
$$

The following lemma is immediate.

Lemma 2.2. Let $A=\left[a_{i j}\right]_{n \times n}$ and $B=\left[b_{i j}\right]_{n \times n}$. Then

(1) $S\left(A^{T}\right)=S(A)$ and $S(\alpha A)=\alpha S(A)$ for every $\alpha \in \mathbb{R}$;

(2) $S(A+B)=S(A)+S(B)$.

Lemma 2.3. Let $G$ be a $(p, q)$-graph, and let $\operatorname{con}(G)$ be a $\left(p, q^{\prime}\right)$-graph. Let $A, B, K$ be the adjacency matrices of $G, \operatorname{con}(G), K_{p}$, respectively. Then

(1) $S(A)=2 q$;

(2) $S\left(A^{2}\right)=M_{1}(G)$;

(3) $S(A B)=N_{1}(G)$;

(4) $S\left(A^{3}\right)=2 M_{2}(G)$;

(5) $S(A K)=2 q(p-1)$.

(6) $S(A B A)=2 N_{2}(G)$.

Proof. For (1), we have

$$
S(A)=\sum_{1 \leq i, j \leq p} a_{i j}=\sum_{i=1}^{p} \sum_{j=1}^{p} a_{i j}=\sum_{i=1}^{p} d_{v_{i}}=2 q
$$


For (2), we have

$$
\begin{aligned}
S\left(A^{2}\right) & =\sum_{1 \leq i, j \leq p} a_{i j}^{(2)}=\sum_{1 \leq i, j \leq p} \sum_{k=1}^{p} a_{i k} a_{k j} \\
& =\sum_{k=1}^{p} \sum_{i=1}^{p} a_{i k} \sum_{j=1}^{p} a_{k j}=\sum_{k=1}^{p} d_{v_{k}} d_{v_{k}}=M_{1}(G)
\end{aligned}
$$

For (3), we have

$$
\begin{aligned}
S(A B) & =\sum_{1 \leq i, j \leq p} \sum_{k=1}^{p} a_{i k} b_{k j} \\
& =\sum_{k=1}^{p} \sum_{i=1}^{p} a_{i k} \sum_{j=1}^{p} b_{k j}=\sum_{k=1}^{p} d_{v_{k}} d_{\text {con } G} v_{k}=N_{1}(G) .
\end{aligned}
$$

For (4), we have

$$
\begin{aligned}
M_{2}(G) & =\sum_{v_{i} v_{j} \in E(G)} d_{v_{i}} d_{v_{j}}=\frac{1}{2} \sum_{1 \leq i, j \leq p} d_{v_{i}} d_{v_{j}} a_{i j} \\
& =\frac{1}{2} \sum_{i=1}^{p} \sum_{j=1}^{p}\left(\sum_{k=1}^{p} a_{k i}\right)\left(\sum_{s=1}^{p} a_{j s}\right) a_{i j} \\
& =\frac{1}{2} \sum_{k=1}^{p} \sum_{j=1}^{p} \sum_{s=1}^{p} a_{j s} \sum_{i=1}^{p} a_{k i} a_{i j} .
\end{aligned}
$$

Since $\sum_{i=1}^{p} a_{k i} a_{i j}$ is the entry $t_{k j}$ of matrix $A^{2}$, it follows that

$$
\begin{aligned}
M_{2}(G) & =\frac{1}{2} \sum_{k=1}^{p} \sum_{j=1}^{p} \sum_{s=1}^{p} a_{j s} \sum_{i=1}^{p} a_{k i} a_{i j} \\
& =\frac{1}{2} \sum_{k=1}^{p} \sum_{s=1}^{p} \sum_{j=1}^{p} t_{k j} a_{j s}=\frac{1}{2} \sum_{k=1}^{p} \sum_{s=1}^{p} a_{k s}^{(3)}=\frac{1}{2} S\left(A^{3}\right) .
\end{aligned}
$$

For (5), we have

$$
\begin{aligned}
S(A K) & =\sum_{1 \leq i, j \leq p} \sum_{r=1}^{p} a_{i r} k_{r j} \\
& =\sum_{r=1}^{p} \sum_{i=1}^{p} a_{i r} \sum_{j=1}^{p} k_{r j}=\sum_{r=1}^{p} d_{v_{r}}(p-1)=2 q(p-1) .
\end{aligned}
$$

For (6), we have

$$
\begin{aligned}
N_{2}(G) & =\sum_{v_{i} v_{j} \in E(\operatorname{con} G)} d_{v_{i}} d_{v_{j}}=\frac{1}{2} \sum_{1 \leq i, j \leq p} d_{v_{i}} d_{v_{j}} b_{i j} \\
& =\frac{1}{2} \sum_{i=1}^{p} \sum_{j=1}^{p}\left(\sum_{k=1}^{p} a_{k i}\right)\left(\sum_{s=1}^{p} a_{s j}\right) b_{i j} \\
& =\frac{1}{2} \sum_{k=1}^{p} \sum_{s=1}^{p} \sum_{j=1}^{p} a_{s j} \sum_{i=1}^{p} a_{k i} b_{i j} .
\end{aligned}
$$


Since, $\sum_{i=1}^{p} a_{k i} b_{i j}$ is the entry $t_{k j}$ of matrix $A B$, hence

$$
\begin{aligned}
N_{2}(G) & =\frac{1}{2} \sum_{k=1}^{p} \sum_{s=1}^{p} \sum_{j=1}^{p} a_{s j} \sum_{i=1}^{p} a_{k i} b_{i j} \\
& =\frac{1}{2} \sum_{k=1}^{p} \sum_{s=1}^{p} \sum_{j=1}^{p} t_{k j} a_{j s}=\frac{1}{2} \sum_{k=1}^{p} \sum_{s=1}^{p} f_{k s}=\frac{1}{2} S(A B A),
\end{aligned}
$$

where $\sum_{i=1}^{p} t_{k j} a_{j s}$ is the entry $f_{k s}$ of matrix $A B A$.

The following result for classical distance are from the book [18].

Lemma 2.4. [18] Let $(u, v)$ and $\left(u^{\prime}, v^{\prime}\right)$ be two vertices of $G_{1} \otimes G_{2}$. Then

$$
d_{G_{1} \bowtie G_{2}}\left((u, v),\left(u^{\prime}, v^{\prime}\right)\right)=\max \left\{d_{G_{1}}\left(u, u^{\prime}\right), d_{G_{2}}\left(v, v^{\prime}\right)\right\} .
$$

For $G_{2}=K_{p}$, the following result is immediate.

Corollary 2.5. Let $K_{p}$ be a complete graph, and let $(u, v)$ and $\left(u^{\prime}, v^{\prime}\right)$ be two vertices of $G \otimes K_{p}$. Then

$$
d_{G \otimes K_{p}}\left((u, v),\left(u^{\prime}, v^{\prime}\right)\right)= \begin{cases}d_{G}\left(u, u^{\prime}\right) & \text { if } u \neq u^{\prime}, \\ 1 & \text { if } u=u^{\prime} \text { and } v \neq v^{\prime}, \\ 0 & \text { if } u=u^{\prime} \text { and } v=v^{\prime} .\end{cases}
$$

\section{Main results}

In this section, we give our main results and their proofs.

\subsection{Relation between degree distance and Gutman index}

We first define a matrix, which will be used later.

Definition 3.1. Let $G(V, E)$ be a graph with order $n$ and $m$ edges. For $k=1,2, \cdots, \alpha$ where $\alpha$ denotes the diameter of graph $G$, we define

$$
A_{k}=\left[a_{i j}^{k}\right]_{n \times n},
$$

where $a_{i j}^{k}= \begin{cases}1 & d\left(v_{i}, v_{j}\right)=k \\ 0 & \text { otherwise }\end{cases}$

The following results are easily seen.

Observation 3.1. Let $A$ and $D_{G}$ be the adjacency matrix and the distance matrix of a graph $G$, respectively. Then

(1) $A_{1}=A$;

(2) $D_{G}=A_{1}+2 A_{2}+\cdots+\alpha A_{\alpha}$;

(3) $A_{1}+A_{2}+\cdots+A_{\alpha}=K$, where $K$ is the adjacency matrix complete graph $K_{n}$;

(4) if $\operatorname{diam}(G)=2$ then $D_{G}=A+2 \bar{A}$. 
Lemma 3.2. Let $G$ be a graph containing no triangles, and let $A, B, K$ be the adjacency matrix of $G, \operatorname{con}(G), K_{n}$, respectively. Then

(1) for every $u, v \in V(G), d_{G}(u, v)=2$ if and only if $u v \in E(\operatorname{con}(G))$;

(2) if $\operatorname{diam}(G)=3$ then $D_{G}=3 K-2 A-B$.

Proof. (1) Suppose $d_{G}(u, v)=2$. Then there exists a vertex $x \in V(G)$ such that $x \notin\{u, v\}$ and $u x, x v \in E(G)$, and hence $N(u) \cap N(v) \neq \emptyset$. Therefore, we have $u v \in E(\operatorname{con}(G))$. Conversely, we suppose $u v \in E(\operatorname{con}(G))$. Then $N(u) \cap N(v) \neq \emptyset$, and hence there exists a vertex $x \in N(u) \cap N(v)$. Note that $u x, x v \in E(G)$. Therefore, $d_{(u, v)} \leq 2$. If $d(u, v)=1$, then we have a triangle, a contradiction. So $d_{G}(u, v)=2$, as desired.

(2) From Observation 3.1, we have $D_{G}=A_{1}+2 A_{2}+3 A_{3}$. Note that $A_{1}=A, A_{2}=B$ and $A+B+A_{3}=K$. Therefore, $D_{G}=3 K-2 A-B$.

Lemma 3.3. Let $G(p, q)$ be a graph, and let $A, D_{G}$ be the adjacency matrix and the distance matrix of a graph $G$, respectively. Then

(1) $S\left(A D_{G}\right)=D D(G)$;

(2) if $\operatorname{diam}(G)=2$ then $D D(G)=4(p-1) q-M_{1}(G)$;

(3) if $\operatorname{diam}(G)=3$ and $G$ has no triangles, then

$$
D D(G)=6 q(p-1)-2 M_{1}(G)-N_{1}(G)
$$

Proof. (1) Since

$$
\begin{aligned}
S\left(A D_{G}\right) & =\sum_{i=1}^{p} \sum_{j=1}^{p} \sum_{k=1}^{p} a_{i k} d_{k j}=\sum_{1 \leq j, k \leq p} \sum_{i=1}^{p} a_{i k} d\left(v_{k}, v_{j}\right) \\
& =\sum_{1 \leq j, k \leq p} d\left(v_{k}\right) d\left(v_{k}, v_{j}\right)
\end{aligned}
$$

and

$$
\begin{aligned}
S\left(D_{G} A\right) & =\sum_{i=1}^{p} \sum_{j=1}^{p} \sum_{k=1}^{p} d_{i k} a_{k j}=\sum_{1 \leq i, k \leq p} d\left(v_{i}, v_{k}\right) \sum_{j=1}^{p} a_{k j} \\
& =\sum_{1 \leq i, k \leq p} d\left(v_{i}, v_{k}\right) d\left(v_{k}\right)=\sum_{1 \leq j, k \leq p} d\left(v_{k}, v_{j}\right) d\left(v_{j}\right),
\end{aligned}
$$

it follows that

$$
\begin{aligned}
2 S\left(A D_{G}\right) & =S\left(A D_{G}\right)+S\left(\left(A D_{G}\right)^{T}\right)=S\left(A D_{G}\right)+S\left(D_{G} A\right) \\
& =\sum_{1 \leq j, k \leq p} d\left(v_{k}, v_{j}\right)\left[d\left(v_{k}\right)+d\left(v_{j}\right)\right] \\
& =2 \sum_{\left\{v_{k}, v_{j}\right\} \subseteq V(G)} d\left(v_{k}, v_{j}\right)\left[d\left(v_{k}\right)+d\left(v_{j}\right)\right]=2 D D(G) .
\end{aligned}
$$

For (2), we have

$$
\begin{aligned}
D D(G) & =S\left(A D_{G}\right)=S(A(A+2 \bar{A})) \\
& =2 S(A(A+\bar{A}))-S\left(A^{2}\right) \\
& =2 S(A K)-S\left(A^{2}\right)=4(p-1) q-M_{1}(G)
\end{aligned}
$$


For (3), we have

$$
\begin{aligned}
D D(G) & =S\left(A D_{G}\right) \\
& =S(A(3 K-2 A-B))=3 S(A K)-2 S\left(A^{2}\right)-S(A B) \\
& =6 q(p-1)-2 M_{1}(G)-N_{1}(G) .
\end{aligned}
$$

Lemma 3.4. Let $G$ be $a(p, q)$-graph and $A$ be the adjacency matrix of $G$. Then $G u t(G)=\frac{1}{2} S\left(A D_{G} A\right)$

\section{Proof.}

$$
\begin{aligned}
S\left(A D_{G} A\right) & =\sum_{i=1}^{p} \sum_{j=1}^{p} \sum_{k=1}^{p} a_{i k} \sum_{s=1}^{p} d_{k s} a_{s j} \\
& =\sum_{k=1}^{p} \sum_{s=1}^{p} \sum_{i=1}^{p} a_{i k} \sum_{j=1}^{p} a_{s j} d_{k s} \\
& =\sum_{k=1}^{p} \sum_{s=1}^{p} d_{G}\left(v_{k}\right) \cdot d_{G}\left(v_{s}\right) \cdot d\left(v_{k}, v_{s}\right) \\
& =2 \sum_{\left\{v_{k}, v_{s}\right\} \subseteq V} d_{G}\left(v_{k}\right) d_{G}\left(v_{s}\right) d\left(v_{k}, v_{s}\right)=2 G u t(G) .
\end{aligned}
$$

Corollary 3.5. Let $G(p, q)$ be a graph, then

$$
\frac{\delta}{2} \leq \frac{G u t(G)}{D D(G)} \leq \frac{\Delta}{2}
$$

Proof. Since, $S\left(A D_{G}\right)=D D(G)$ and $G u t(G)=\frac{1}{2} S\left(A D_{G} A\right)$, hence

$$
\begin{aligned}
2 G u t(G)-D D(G) & =S\left(A D_{G} A\right)-S\left(A D_{G}\right) \\
& =S\left(A D_{G}(A-I)\right) \\
& =\sum_{1 \leq i, j \leq p} \sum_{k=1}^{p} t_{i k}\left(a_{k j}-1_{k j}\right) \\
& =\sum_{1 \leq i, k \leq p} t_{i k}\left(d_{G}\left(v_{k}\right)-1\right) .
\end{aligned}
$$

Therefore,

$$
(\delta-1) \sum_{1 \leq i, k \leq p} t_{i k} \leq 2 G u t(G)-D D(G) \leq(\Delta-1) \sum_{1 \leq i, k \leq p} t_{i k}
$$

Hence,

$$
(\delta-1) S\left(A D_{G}\right) \leq 2 G u t(G)-D D(G) \leq(\Delta-1) S\left(A D_{G}\right)
$$

Thus,

$$
\delta D D(G) \leq 2 G u t(G) \leq \Delta D D(G)
$$

that is

$$
\frac{\delta}{2} \leq \frac{G u t(G)}{D D(G)} \leq \frac{\Delta}{2}
$$




\subsection{For degree distance}

In this subsection, we study the degree distance of strong product graphs. We first begin with an easy case.

Theorem 3.6. Let $G$ be a connected graph with $p_{1}$ vertices and $q_{1}$ edges, and $K_{p}$ be a complete graph with order $p$. Then

$$
D D\left(G \otimes K_{p}\right)=p^{3} D D(G)+2 p^{2}(p-1)\left[W(G)+q_{1}\right]+p_{1} p(p-1)^{2} .
$$

Proof. Let $V(G)=V_{1}$ and $V\left(K_{p}\right)=V_{2}$. From the definition of strong product and Corollary 2.5, we have

$$
\begin{aligned}
& D D\left(G \otimes K_{p}\right) \\
& =\sum_{\{(a, b),(c, d)\} \subseteq V_{1} \times V_{2}}\left[d_{G \bowtie K_{p}}(a, b)+d_{G \otimes K_{p}}(c, d)\right] d_{G \bowtie K_{p}}[(a, b),(c, d)] \\
& =\sum_{\{(a, b),(c, d)\} \subseteq V_{1} \times V_{2}}\left[d_{G}(a)+d_{K_{p}}(b)+d_{G}(a) d_{K_{p}}(b)+d_{G}(c)+d_{K_{p}}(d)+d_{G}(c) d_{K_{p}}(d)\right] \\
& =\sum_{\{(a, b),(c, d)\} \subseteq V_{1} \times V_{2}, a \neq c}\left[d_{G}(a)+p-1+d_{G}(a)(p-1)+d_{G}(c)+p-1+d_{G}(c)(p-1)\right] \cdot d_{G}(a, c) \\
& +\sum_{\{(a, b),(c, d)\} \subseteq V_{1} \times V_{2}, a=c}\left[d_{G}(a)+p-1+d_{G}(a)(p-1)+d_{G}(c)+p-1+d_{G}(c)(p-1)\right] \cdot 1 \\
& =\sum_{\{(a, b),(c, d)\} \subseteq V_{1} \times V_{2}, a \neq c}\left[p\left(d_{G}(a)+d_{G}(c)\right)+2(p-1)\right] d_{G}(a, c) \\
& +\sum_{\{(a, b),(c, d)\} \subseteq V_{1} \times V_{2}, a=c}\left[2 p d_{G}(a)+2(p-1)\right] \\
& =p \sum_{\{(a, b),(c, d)\} \subseteq V_{1} \times V_{2}, a \neq c}\left[d_{G}(a)+d_{G}(c)\right] d_{G}(a, c)+2(p-1) \sum_{\{(a, b),(c, d)\} \subseteq V_{1} \times V_{2}, a \neq c} d_{G}(a, c) \\
& +2 p \sum_{\{(a, b),(c, d)\} \subseteq V_{1} \times V_{2}, a=c} d_{G}(a)+2(p-1) \sum_{\{(a, b),(c, d)\} \subseteq V_{1} \times V_{2}, a=c} 1 \\
& =p^{3} D D(G)+2 p^{2}(p-1) W(G)+2 p \cdot \frac{p(p-1)}{2} \cdot 2 q_{1}+2 p_{1}(p-1) \cdot \frac{p(p-1)}{2} \\
& =p^{3} D D(G)+2 p^{2}(p-1)\left[W(G)+q_{1}\right]+p_{1} p(p-1)^{2} .
\end{aligned}
$$

For the strong product of two general graphs, we have the following.

Theorem 3.7. Let $G_{1}$ be a connected graph with $p_{1}$ vertices and $q_{1}$ edges, and $G_{2}$ be a connected graph 
with $p_{2}$ vertices and $q_{2}$ edges. Then

$$
\begin{aligned}
& \max \left\{D D\left(G_{1}\right)\left[2 p_{2} q_{2}+p_{2}^{2}\right]+4 p_{2} q_{2} W\left(G_{1}\right)+2 p_{2}\left(p_{2}-1\right) q_{1} W\left(G_{2}\right)+D D\left(G_{2}\right)\left(2 q_{1}+p_{1}\right),\right. \\
& \left.D D\left(G_{2}\right)\left[2 p_{1} q_{1}+p_{1}^{2}\right]+4 p_{1} q_{1} W\left(G_{2}\right)+2 p_{1}\left(p_{1}-1\right) q_{2} W\left(G_{1}\right)+D D\left(G_{1}\right)\left(2 q_{2}+p_{2}\right)\right\} \\
\leq & D D\left(G_{1} \otimes G_{2}\right) \leq\left(2 q_{2}+p_{2}\right)\left(p_{2}+1\right) D D\left(G_{1}\right)+q_{2}\left(4 p_{2}+2 p_{1}^{2}-2 p_{1}\right) W\left(G_{1}\right) \\
& +\left(2 q_{1}+p_{1}\right)\left(p_{1}+1\right) D D\left(G_{2}\right)+q_{1}\left(4 p_{1}+2 p_{2}^{2}-2 p_{2}\right) W\left(G_{2}\right) .
\end{aligned}
$$

Moreover, the lower bound is sharp.

In particular, if $G$ be a connected graph with $p$ vertices and $q$ edges, then

$$
\begin{aligned}
& (2 q+p)(p+1) D D(G)+2 p q(p+1) W(G) \\
\leq & D D(G \otimes G) \leq 2\{(2 q+p)(p+1) D D(G)+2 p q(p+1) W(G)\} .
\end{aligned}
$$

Proof. From Lemma 2.4 and the definition of degree distance, we have

$$
\begin{aligned}
& D D\left(G_{1} \otimes G_{2}\right)=\sum_{\{(a, b),(c, d)\} \subseteq V_{1} \times V_{2}}\left[d_{G_{1} \otimes G_{2}}(a, b)+d_{G_{1} \otimes G_{2}}(c, d)\right] d_{G_{1} \otimes G_{2}}[(a, b),(c, d)] \\
& =\sum_{\{(a, b),(c, d)\} \subseteq V_{1} \times V_{2}}\left[d_{G_{1}}(a)+d_{G_{2}}(b)+d_{G_{1}}(a) d_{G_{2}}(b)+d_{G_{1}}(c)+d_{G_{2}}(d)+d_{G_{1}}(c) d_{G_{2}}(d)\right] \\
& \cdot \max \left\{d_{G_{1}}(a, c), d_{G_{2}}(b, d)\right\} \\
& \geq \max \left\{\sum_{\substack{\{(a, b),(c, d)\} \subseteq V_{1} \times V_{2} \\
a \neq c}}\left[d_{G_{1}}(a)+d_{G_{2}}(b)+d_{G_{1}}(a) d_{G_{2}}(b)+d_{G_{1}}(c)+d_{G_{2}}(d)+d_{G_{1}}(c) d_{G_{2}}(d)\right] d_{G_{1}}(a, c)\right. \\
& +\sum_{\{(a, b),(c, d)\} \subseteq V_{1} \times V_{2}}\left[d_{G_{1}}(a)+d_{G_{2}}(b)+d_{G_{1}}(a) d_{G_{2}}(b)+d_{G_{1}}(c)+d_{G_{2}}(d)+d_{G_{1}}(c) d_{G_{2}}(d)\right] d_{G_{2}}(b, d), \\
& \sum_{\substack{\{(a, b),(c, d)\} \subseteq V_{1} \times V_{2} \\
b \neq d}}\left[d_{G_{1}}(a)+d_{G_{2}}(b)+d_{G_{1}}(a) d_{G_{2}}(b)+d_{G_{1}}(c)+d_{G_{2}}(d)+d_{G_{1}}(c) d_{G_{2}}(d)\right] d_{G_{2}}(b, d) \\
& \left.+\sum_{\substack{\{(a, b),(c, d)\} \subseteq V_{1} \times V_{2} \\
b=d}}\left[d_{G_{1}}(a)+d_{G_{2}}(b)+d_{G_{1}}(a) d_{G_{2}}(b)+d_{G_{1}}(c)+d_{G_{2}}(d)+d_{G_{1}}(c) d_{G_{2}}(d)\right] d_{G_{1}}(a, c)\right\}
\end{aligned}
$$




$$
\begin{aligned}
& =\max \left\{\sum_{\substack{\{(a, b),(c, d)\} \subseteq V_{1} \times V_{2} \\
a \neq c}}\left[d_{G_{1}}(a)+d_{G_{1}}(c)\right] d_{G_{1}}(a, c)+\sum_{\substack{\{(a, b),(c, d)\} \subseteq V_{1} \times V_{2} \\
a \neq c}}\left[d_{G_{2}}(b)+d_{G_{2}}(d)\right] d_{G_{1}}(a, c)\right.
\end{aligned}
$$

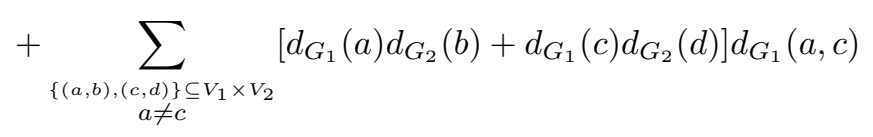

$$
\begin{aligned}
& +\sum_{\{(a, b),(a, d)\} \subseteq V_{1} \times V_{2}}\left[2 d_{G_{1}}(a)+\left(d_{G_{1}}(a)+1\right)\left(d_{G_{2}}(b)+d_{G_{2}}(d)\right)\right] d_{G_{2}}(b, d),
\end{aligned}
$$

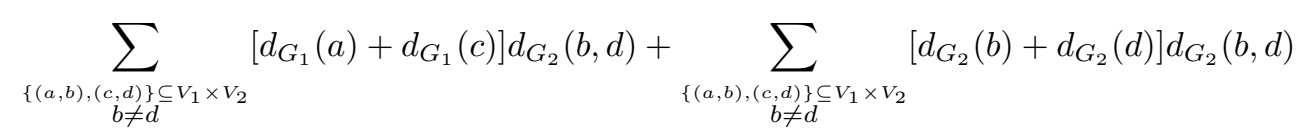

$$
\begin{aligned}
& +\sum_{\substack{\{(a, b),(c, d)\} \subseteq V_{1} \times V_{2} \\
b \neq d}}\left[d_{G_{1}}(a) d_{G_{2}}(b)+d_{G_{1}}(c) d_{G_{2}}(d)\right] d_{G_{2}}(b, d) \\
& \left.+\sum_{\{(a, b),(c, b)\} \subseteq V_{1} \times V_{2}}\left[2 d_{G_{2}}(b)+\left(d_{G_{2}}(b)+1\right)\left(d_{G_{1}}(a)+d_{G_{1}}(c)\right)\right] d_{G_{1}}(a, c)\right\} \\
& =\max \left\{p_{2}^{2} D D\left(G_{1}\right)+4 p_{2} q_{2} W\left(G_{1}\right)+\sum_{\{(a, b),(c, d)\} \subseteq V_{1} \times V_{2}}\left[d_{G_{1}}(a) d_{G_{2}}(b)+d_{G_{1}}(c) d_{G_{2}}(d)\right] d_{G_{1}}(a, c)\right. \\
& +2 p_{2}\left(p_{2}-1\right) q_{1} W\left(G_{2}\right)+D D\left(G_{2}\right)\left(2 q_{1}+p_{1}\right) \\
& p_{1}^{2} D D\left(G_{2}\right)+4 p_{1} q_{1} W\left(G_{2}\right)+\sum_{\{(a, b),(c, d)\} \subseteq V_{1} \times V_{2}}\left[d_{G_{1}}(a) d_{G_{2}}(b)+d_{G_{1}}(c) d_{G_{2}}(d)\right] d_{G_{2}}(b, d) \\
& \left.+2 p_{1}\left(p_{1}-1\right) q_{2} W\left(G_{1}\right)+D D\left(G_{1}\right)\left(2 q_{2}+p_{2}\right)\right\}
\end{aligned}
$$

Since

$$
\begin{aligned}
& \sum_{\{(a, b),(c, d)\} \subseteq V_{1} \times V_{2}}\left[d_{G_{1}}(a) d_{G_{2}}(b)+d_{G_{1}}(c) d_{G_{2}}(d)\right] d_{G_{1}}(a, c) \\
= & \sum_{\{(a, b),(c, d)\} \subseteq V_{1} \times V_{2}}\left[d_{G_{1}}(a) d_{G_{2}}(b) \cdot d_{G_{1}}(a, c)\right]+\sum_{\{(a, b),(c, d)\} \subseteq V_{1} \times V_{2}}\left[d_{G_{1}}(c) d_{G_{2}}(d) \cdot d_{G_{1}}(a, c)\right]
\end{aligned}
$$




$$
\begin{aligned}
& =\sum_{\{a, c\} \subseteq V_{1}} d_{G_{1}}(a) d_{G_{1}}(a, c) \cdot \sum_{\{b, d\} \subseteq V_{2}} d_{G_{2}}(b)+\sum_{\{a, c\} \subseteq V_{1}} d_{G_{1}}(c) d_{G_{1}}(a, c) \cdot \sum_{\{b, d\} \subseteq V_{2}} d_{G_{2}}(d) \\
& =2 p_{2} q_{2} \cdot \sum_{\{a, c\} \subseteq V_{1}} d_{G_{1}}(a) d_{G_{1}}(a, c)+2 p_{2} q_{2} \cdot \sum_{\{a, c\} \subseteq V_{1}} d_{G_{1}}(c) d_{G_{1}}(a, c) \\
& =2 p_{2} q_{2}\left(\sum_{\{a, c\} \subseteq V_{1}}\left[d_{G_{1}}(a)+d_{G_{1}}(c)\right] d_{G_{1}}(a, c)\right) \\
& =2 p_{2} q_{2} \cdot D D\left(G_{1}\right)
\end{aligned}
$$

and similarly

$$
\begin{gathered}
\sum_{\{(a, b),(c, d)\} \subseteq V_{1} \times V_{2}}\left[d_{G_{1}}(a) d_{G_{2}}(b)+d_{G_{1}}(c) d_{G_{2}}(d)\right] d_{G_{2}}(b, d) \\
=2 p_{1} q_{1}\left(\sum_{\{b, d\} \subseteq V_{2}}\left[d_{G_{2}}(b)+d_{G_{2}}(d)\right] d_{G_{2}}(b, d)\right)=2 p_{1} q_{1} D D\left(G_{2}\right),
\end{gathered}
$$

it follows that

$$
\begin{aligned}
& D D\left(G_{1} \otimes G_{2}\right) \\
\geq & \max \left\{D D\left(G_{1}\right)\left[2 p_{2} q_{2}+p_{2}^{2}\right]+4 p_{2} q_{2} W\left(G_{1}\right)+2 p_{2}\left(p_{2}-1\right) q_{1} W\left(G_{2}\right)+D D\left(G_{2}\right)\left(2 q_{1}+p_{1}\right),\right. \\
& \left.D D\left(G_{2}\right)\left[2 p_{1} q_{1}+p_{1}^{2}\right]+4 p_{1} q_{1} W\left(G_{2}\right)+2 p_{1}\left(p_{1}-1\right) q_{2} W\left(G_{1}\right)+D D\left(G_{1}\right)\left(2 q_{2}+p_{2}\right)\right\} .
\end{aligned}
$$

Also, we have

$$
\begin{aligned}
& D D\left(G_{1} \otimes G_{2}\right) \\
& =\sum_{\{(a, b),(c, d)\} \subseteq V_{1} \times V_{2}}\left[d_{G_{1} \bowtie G_{2}}(a, b)+d_{G_{1} \bowtie G_{2}}(c, d)\right] d_{G_{1} \bowtie G_{2}}[(a, b),(c, d)] \\
& =\sum_{\{(a, b),(c, d)\} \subseteq V_{1} \times V_{2}}\left[d_{G_{1}}(a)+d_{G_{2}}(b)+d_{G_{1}}(a) d_{G_{2}}(b)+d_{G_{1}}(c)+d_{G_{2}}(d)+d_{G_{1}}(c) d_{G_{2}}(d)\right] \\
& \cdot \max \left\{d_{G_{1}}(a, c), d_{G_{2}}(b, d)\right\} \\
& \leq \sum_{\{(a, b),(c, d)\} \subseteq V_{1} \times V_{2}}\left[d_{G_{1}}(a)+d_{G_{2}}(b)+d_{G_{1}}(a) d_{G_{2}}(b)+d_{G_{1}}(c)+d_{G_{2}}(d)+d_{G_{1}}(c) d_{G_{2}}(d)\right] d_{G_{1}}(a, c) \\
& +\sum_{\{(a, b),(c, d)\} \subseteq V_{1} \times V_{2}}\left[d_{G_{1}}(a)+d_{G_{2}}(b)+d_{G_{1}}(a) d_{G_{2}}(b)+d_{G_{1}}(c)+d_{G_{2}}(d)+d_{G_{1}}(c) d_{G_{2}}(d)\right] d_{G_{2}}(b, d) \\
& =\left(2 q_{2}+p_{2}\right)\left(p_{2}+1\right) D D\left(G_{1}\right)+q_{2}\left(4 p_{2}+2 p_{1}^{2}-2 p_{1}\right) W\left(G_{1}\right) \\
& +\left(2 q_{1}+p_{1}\right)\left(p_{1}+1\right) D D\left(G_{2}\right)+q_{1}\left(4 p_{1}+2 p_{2}^{2}-2 p_{2}\right) W\left(G_{2}\right) \text {. }
\end{aligned}
$$

To show the sharpness of the lower bounds of Theorem 3.7, we consider the following example.

Example 1. Let $G$ be a complete graph of order $n$. If $n=2$, then $G=K_{2}$ and $G \otimes G=K_{4}$, and hence $D D(G \otimes G)=36=(2 q+p)(p+1) D D(G)+2 p q(p+1) W(G)$. If $n=3$, then $G=K_{3}$ and $G \otimes G=K_{9}$, and hence $D D(G \otimes G)=576=(2 q+p)(p+1) D D(G)+2 p q(p+1) W(G)$. From the proof of Theorem 3.7 , one can check that $K_{n} \otimes K_{n}$ is an sharp example of the lower bound. 


\subsection{For Gutman index}

In this subsection, we study the Gutman index of strong product graphs. We first begin with an easy case.

Theorem 3.8. Let $G$ be a connected graph with $p_{1}$ vertices and $q_{1}$ edges, and $K_{p}$ be a complete graph with $p$ vertices. Then

$$
\begin{aligned}
G u t\left(G \otimes K_{p}\right) & =p^{4} G u t(G)+p^{3}(p-1) D D(G)+p^{2}(p-1)^{2} \cdot W(G) \\
& +\frac{p^{3}(p-1)}{2} M_{1}(G)+\frac{p(p-1)^{3}}{2} p_{1}+2 p^{2}(p-1)^{2} q_{1}
\end{aligned}
$$

Proof. Let $V(G)=V_{1}$ and $V\left(K_{p}\right)=V_{2}$. From the definition of strong product and Corollary 2.5, we have

$$
\begin{aligned}
& G u t\left(G \otimes K_{p}\right) \\
& =\sum_{\{(a, b),(c, d)\} \subseteq V_{1} \times V_{2}} d_{G \otimes K_{p}}(a, b) \cdot d_{G \otimes K_{p}}(c, d) \cdot d_{G \otimes K_{p}}[(a, b),(c, d)] \\
& =\sum_{\{(a, b),(c, d)\} \subseteq V_{1} \times V_{2}}\left[d_{G}(a)+d_{K_{p}}(b)+d_{G}(a) d_{K_{p}}(b)\right] \cdot\left[d_{G}(c)+d_{K_{p}}(d)+d_{G}(c) d_{K_{p}}(d)\right] \\
& \cdot d_{G \otimes K_{p}}[(a, b),(c, d)] \\
& =\sum_{\{(a, b),(c, d)\} \subseteq V_{1} \times V_{2}, a \neq c}\left[p d_{G}(a)+p-1\right] \cdot\left[p d_{G}(c)+p-1\right] \cdot d_{G}(a, c) \\
& +\sum_{\{(a, b),(c, d)\} \subseteq V_{1} \times V_{2}, a=c}\left[p d_{G}(a)+p-1\right] \cdot\left[p d_{G}(a)+(p-1)\right] \cdot 1 \\
& =p^{2} \sum_{\{(a, b),(c, d)\} \subseteq V_{1} \times V_{2}, a \neq c} d_{G}(a) d_{G}(c) d_{G}(a, c) \\
& +p(p-1) \sum_{\{(a, b),(c, d)\} \subseteq V_{1} \times V_{2}, a \neq c}\left[d_{G}(a)+d_{G}(c)\right] d_{G}(a, c) \\
& +(p-1)^{2} \sum_{\{(a, b),(c, d)\} \subseteq V_{1} \times V_{2}, a \neq c} d_{G}(a, c)+p^{2} \sum_{\{(a, b),(c, d)\} \subseteq V_{1} \times V_{2}, a=c} d_{G}^{2}(a) \\
& +\sum_{\{(a, b),(c, d)\} \subseteq V_{1} \times V_{2}, a=c}(p-1)^{2}+2 p(p-1) \sum_{\{(a, b),(c, d)\} \subseteq V_{1} \times V_{2}, a=c} d_{G}(a) \\
& =p^{4} G u t(G)+p^{3}(p-1) D D(G)+p^{2}(p-1)^{2} \cdot W(G) \\
& +\frac{p^{3}(p-1)}{2} M_{1}(G)+\frac{p(p-1)^{3}}{2} p_{1}+2 p^{2}(p-1)^{2} q_{1} .
\end{aligned}
$$

For the strong product of two general graphs, we have the following. 
Theorem 3.9. Let $G_{1}$ be a connected graph with $p_{1}$ vertices and $q_{1}$ edges, and $G_{2}$ be a connected graph with $p_{2}$ vertices and $q_{1}$ edges. Then

$$
\begin{aligned}
& \max \left\{G u t\left(G_{1}\right)\left(p_{2}^{2}+4 p_{2} q_{2}+4 q_{2}^{2}\right)+\left(2 p_{2} q_{2}+4 q_{2}^{2}\right) D D\left(G_{1}\right)+4 q_{2}^{2} W\left(G_{1}\right)\right. \\
& +M_{1}\left(G_{1}\right) W\left(G_{2}\right)+\left[2 q_{1}+M_{1}\left(G_{1}\right)\right] D D\left(G_{2}\right)+\left[p_{1}+4 q_{1}+M_{1}\left(G_{1}\right)\right] G u t\left(G_{2}\right), \\
& \operatorname{Gut}\left(G_{2}\right)\left(p_{1}^{2}+4 p_{1} q_{1}+4 q_{1}^{2}\right)+\left(2 p_{1} q_{1}+4 q_{1}^{2}\right) D D\left(G_{2}\right)+4 q_{1}^{2} W\left(G_{2}\right) \\
& \left.+M_{2}\left(G_{2}\right) W\left(G_{1}\right)+\left[2 q_{2}+M_{2}\left(G_{2}\right)\right] D D\left(G_{1}\right)+\left[p_{2}+4 q_{2}+M_{2}\left(G_{2}\right)\right] G u t\left(G_{1}\right)\right\} \\
\leq & G u t\left(G_{1} \otimes G_{2}\right) \\
\leq & G u t\left(G_{1}\right)\left[p_{2}^{2}+4 p_{2} q_{2}+4 q_{2}^{2}+p_{2}+4 q_{2}+M_{2}\left(G_{2}\right)\right]+\left[2 p_{2} q_{2}+4 q_{2}^{2}+2 q_{2}+M_{2}\left(G_{2}\right)\right] D D\left(G_{1}\right) \\
& +\left[4 q_{2}^{2}+M_{2}\left(G_{2}\right)\right] W\left(G_{1}\right)+G u t\left(G_{2}\right)\left[p_{1}^{2}+4 p_{1} q_{1}+4 q_{1}^{2}+p_{1}+4 q_{1}+M_{1}\left(G_{1}\right)\right] \\
& +\left[2 p_{1} q_{1}+4 q_{1}^{2}+2 q_{1}+M_{1}\left(G_{1}\right)\right] D D\left(G_{2}\right)+\left[4 q_{1}^{2}+M_{1}\left(G_{1}\right)\right] W\left(G_{2}\right) .
\end{aligned}
$$

In particular, if $G$ be a connected graph with $p$ vertices and $q$ edges, then

$$
\begin{aligned}
& G u t(G)\left[p^{2}+4 p q+4 q^{2}+p+4 q\right]+\left[2 p q+4 q^{2}+2 q+M_{1}(G)\right] D D(G)+\left[4 q^{2}+M_{1}(G)\right] W(G) \\
\leq & G u t(G \otimes G) \\
\leq & 2\left\{G u t(G)\left[p^{2}+4 p q+4 q^{2}+p+4 q\right]+\left[2 p q+4 q^{2}+2 q+M_{1}(G)\right] D D(G)+\left[4 q^{2}+M_{1}(G)\right] W(G)\right\}
\end{aligned}
$$

Proof. Let $V\left(G_{1}\right)=V_{1}$ and $V\left(G_{2}\right)=V_{2}$. From the definition of strong product and Lemma 2.4, we have

$G u t\left(G_{1} \otimes G_{2}\right)$

$$
\begin{aligned}
& =\sum_{\{(a, b),(c, d)\} \subseteq V_{1} \times V_{2}} d_{G_{1} \bowtie G_{2}}(a, b) \cdot d_{G_{1} \bowtie G_{2}}(c, d) \cdot d_{G_{1} \bowtie G_{2}}[(a, b),(c, d)] \\
& =\sum_{\{(a, b),(c, d)\} \subseteq V_{1} \times V_{2}}\left[d_{G_{1}}(a)+d_{G_{2}}(b)+d_{G_{1}}(a) d_{G_{2}}(b)\right] \cdot\left[d_{G_{1}}(c)+d_{G_{2}}(d)+d_{G_{1}}(c) d_{G_{2}}(d)\right] \\
& \cdot \max \left\{d_{G_{1}}(a, c), d_{G_{2}}(b, d)\right\} \\
& \geq \max \left\{\sum_{\substack{\{(a, b),(c, d)\} \subseteq V_{1} \times V_{2} \\
a \neq c}}\left[d_{G_{1}}(a)+d_{G_{2}}(b)+d_{G_{1}}(a) d_{G_{2}}(b)\right] \cdot\left[d_{G_{1}}(c)+d_{G_{2}}(d)+d_{G_{1}}(c) d_{G_{2}}(d)\right] d_{G_{1}}(a, c)\right. \\
& +\sum_{\{(a, b),(c, d)\} \subseteq V_{1} \times V_{2}}\left[d_{G_{1}}(a)+d_{G_{2}}(b)+d_{G_{1}}(a) d_{G_{2}}(b)\right] \cdot\left[d_{G_{1}}(c)+d_{G_{2}}(d)+d_{G_{1}}(c) d_{G_{2}}(d)\right] d_{G_{2}}(b, d), \\
& \sum_{\substack{\{(a, b),(c, d)\} \subseteq V_{1} \times V_{2} \\
b \neq d}}\left[d_{G_{1}}(a)+d_{G_{2}}(b)+d_{G_{1}}(a) d_{G_{2}}(b)\right] \cdot\left[d_{G_{1}}(c)+d_{G_{2}}(d)+d_{G_{1}}(c) d_{G_{2}}(d)\right] d_{G_{2}}(b, d) \\
& \left.+\sum_{\substack{\{(a, b),(c, d)\} \subseteq V_{1} \times V_{2} \\
b=d}}\left[d_{G_{1}}(a)+d_{G_{2}}(b)+d_{G_{1}}(a) d_{G_{2}}(b)\right] \cdot\left[d_{G_{1}}(c)+d_{G_{2}}(d)+d_{G_{1}}(c) d_{G_{2}}(d)\right] d_{G_{1}}(a, c)\right\}
\end{aligned}
$$




$$
\begin{aligned}
& =\max \left\{\sum_{\substack{\{(a, b),(c, d)\} \subseteq V_{1} \times V_{2} \\
a \neq c}}\left[d_{G_{1}}(a)+d_{G_{2}}(b)+d_{G_{1}}(a) d_{G_{2}}(b)\right] \cdot\left[d_{G_{1}}(c)+d_{G_{2}}(d)+d_{G_{1}}(c) d_{G_{2}}(d)\right] d_{G_{1}}(a, c)\right. \\
& +\sum_{\{(a, b),(a, d)\} \subseteq V_{1} \times V_{2}}\left[d_{G_{1}}(a)+d_{G_{2}}(b)+d_{G_{1}}(a) d_{G_{2}}(b)\right] \cdot\left[d_{G_{1}}(a)+d_{G_{2}}(d)+d_{G_{1}}(a) d_{G_{2}}(d)\right] d_{G_{2}}(b, d), \\
& \sum_{\substack{\{(a, b),(c, d)\} \subseteq V_{1} \times V_{2} \\
b \neq d}}\left[d_{G_{1}}(a)+d_{G_{2}}(b)+d_{G_{1}}(a) d_{G_{2}}(b)\right] \cdot\left[d_{G_{1}}(c)+d_{G_{2}}(d)+d_{G_{1}}(c) d_{G_{2}}(d)\right] d_{G_{2}}(b, d) \\
& \left.+\sum_{\{(a, b),(c, b)\} \subseteq V_{1} \times V_{2}}\left[d_{G_{1}}(a)+d_{G_{2}}(b)+d_{G_{1}}(a) d_{G_{2}}(b)\right] \cdot\left[d_{G_{1}}(c)+d_{G_{2}}(b)+d_{G_{1}}(c) d_{G_{2}}(b)\right] d_{G_{1}}(a, c)\right\} \\
& =\max \left\{X_{1}+X_{2}, Y_{1}+Y_{2}\right\}
\end{aligned}
$$

where

$$
\begin{aligned}
X_{1} & =\sum_{\substack{\{(a, b),(c, d)\} \subseteq V_{1} \times V_{2} \\
a \neq c}}\left[d_{G_{1}}(a)+d_{G_{2}}(b)+d_{G_{1}}(a) d_{G_{2}}(b)\right] \cdot\left[d_{G_{1}}(c)+d_{G_{2}}(d)+d_{G_{1}}(c) d_{G_{2}}(d)\right] d_{G_{1}}(a, c), \\
X_{2} & =\sum_{\{(a, b),(a, d)\} \subseteq V_{1} \times V_{2}}\left[d_{G_{1}}(a)+d_{G_{2}}(b)+d_{G_{1}}(a) d_{G_{2}}(b)\right] \cdot\left[d_{G_{1}}(a)+d_{G_{2}}(d)+d_{G_{1}}(a) d_{G_{2}}(d)\right] d_{G_{2}}(b, d), \\
Y_{1} & =\sum_{\substack{\{(a, b),(c, d)\} \subseteq V_{1} \times V_{2} \\
b \neq d}}\left[d_{G_{1}}(a)+d_{G_{2}}(b)+d_{G_{1}}(a) d_{G_{2}}(b)\right] \cdot\left[d_{G_{1}}(c)+d_{G_{2}}(d)+d_{G_{1}}(c) d_{G_{2}}(d)\right] d_{G_{2}}(b, d),
\end{aligned}
$$

and

$$
Y_{2}=\sum_{\{(a, b),(c, b)\} \subseteq V_{1} \times V_{2}}\left[d_{G_{1}}(a)+d_{G_{2}}(b)+d_{G_{1}}(a) d_{G_{2}}(b)\right] \cdot\left[d_{G_{1}}(c)+d_{G_{2}}(b)+d_{G_{1}}(c) d_{G_{2}}(b)\right] d_{G_{1}}(a, c) .
$$

Note that

$$
\begin{aligned}
X_{1}= & \sum_{\substack{\{(a, b),(c, d)\} \subseteq V_{1} \times V_{2} \\
a \neq c}} d_{G_{1}}(a) \cdot d_{G_{1}}(c) \cdot d_{G_{1}}(a, c)+\sum_{\substack{\{(a, b),(c, d)\} \subseteq V_{1} \times V_{2} \\
a \neq c}} d_{G_{1}}(a) \cdot d_{G_{2}}(d) \cdot d_{G_{1}}(a, c) \\
& +\sum_{\substack{\{(a, b),(c, d)\} \subseteq V_{1} \times V_{2} \\
a \neq c}} d_{G_{1}}(a) d_{G_{1}}(c) d_{G_{2}}(d) d_{G_{1}}(a, c)+\sum_{\substack{\{(a, b),(c, d)\} \subseteq V_{1} \times V_{2} \\
a \neq c}} d_{G_{2}}(b) d_{G_{1}}(c) d_{G_{1}}(a, c)
\end{aligned}
$$




$$
\begin{aligned}
& +\sum_{\substack{\{(a, b),(c, d)\} \subseteq V_{1} \times V_{2} \\
a \neq c}} d_{G_{2}}(b) d_{G_{2}}(d) d_{G_{1}}(a, c)+\sum_{\substack{\{(a, b),(c, d)\} \subseteq V_{1} \times V_{2} \\
a \neq c}} d_{G_{2}}(b) d_{G_{1}}(c) d_{G_{2}}(d) d_{G_{1}}(a, c) \\
& +\sum_{\substack{\{(a, b),(c, d)\} \subseteq V_{1} \times V_{2} \\
a \neq c}} d_{G_{1}}(a) d_{G_{2}}(b) d_{G_{1}}(c) d_{G_{1}}(a, c)+\sum_{\substack{\{(a, b),(c, d)\} \subseteq V_{1} \times V_{2} \\
a \neq c}} d_{G_{1}}(a) d_{G_{2}}(b) d_{G_{2}}(d) d_{G_{1}}(a, c) \\
& +\sum_{\substack{\{(a, b),(c, d)\} \subseteq V_{1} \times V_{2} \\
a \neq c}} d_{G_{1}}(a) d_{G_{2}}(b) d_{G_{1}}(c) d_{G_{2}}(d) d_{G_{1}}(a, c) \\
& =p_{2}^{2} G u t\left(G_{1}\right)+4 p_{2} q_{2} G u t\left(G_{1}\right)+4 q_{2}^{2} G u t\left(G_{1}\right)+2 p_{2} q_{2} D D\left(G_{1}\right)+4 q_{2}^{2} D D\left(G_{1}\right)+4 q_{2}^{2} W\left(G_{1}\right) \\
& =\operatorname{Gut}\left(G_{1}\right)\left(p_{2}^{2}+4 p_{2} q_{2}+4 q_{2}^{2}\right)+\left(2 p_{2} q_{2}+4 q_{2}^{2}\right) D D\left(G_{1}\right)+4 q_{2}^{2} W\left(G_{1}\right)
\end{aligned}
$$

and

$$
\begin{aligned}
= & \sum_{\{(a, b),(c, d)\} \subseteq V_{1} \times V_{2}} d_{G_{1}}(a)^{2} \cdot d_{G_{2}}(b, d)+\sum_{\{(a, b),(c, d)\} \subseteq V_{1} \times V_{2}} d_{G_{1}}(a) \cdot d_{G_{2}}(d) \cdot d_{G_{2}}(b, d) \\
& +\sum_{\{(a, b),(c, d)\} \subseteq V_{1} \times V_{2}} d_{G_{1}}(a)^{2} d_{G_{2}}(d) d_{G_{2}}(b, d)+\sum_{\{(a, b),(c, d)\} \subseteq V_{1} \times V_{2}} d_{G_{2}}(b) d_{G_{1}}(a) d_{G_{2}}(b, d) \\
& +\sum_{\{(a, b),(c, d)\} \subseteq V_{1} \times V_{2}} d_{G_{2}}(b) d_{G_{2}}(d) d_{G_{2}}(b, d)+\sum_{\{(a, b),(c, d)\} \subseteq V_{1} \times V_{2}} d_{G_{2}}(b) d_{G_{1}}(a) d_{G_{2}}(d) d_{G_{2}}(b, d) \\
& +\sum_{\{(a, b),(c, d)\} \subseteq V_{1} \times V_{2}} d_{G_{1}}(a)^{2} d_{G_{2}}(b) d_{G_{2}}(b, d)+\sum_{\{(a, b),(c, d)\} \subseteq V_{1} \times V_{2}} d_{G_{1}}(a) d_{G_{2}}(b) d_{G_{2}}(d) d_{G_{2}}(b, d) \\
& +\sum_{\{(a, b),(c, d)\} \subseteq V_{1} \times V_{2}} d_{G_{1}}(a)^{2} d_{G_{2}}(b) d_{G_{2}}(d) d_{G_{2}}(b, d) \\
= & M_{1}\left(G_{1}\right) W\left(G_{2}\right)+2 q_{1} D D\left(G_{2}\right)+M_{1}\left(G_{1}\right) D D\left(G_{2}\right)+p_{1} G u t\left(G_{2}\right)+4 q_{1} G u t\left(G_{2}\right) \\
& +M_{1}\left(G_{1}\right) G u t\left(G_{2}\right) \\
= & M_{1}\left(G_{1}\right) W\left(G_{2}\right)+\left[2 q_{1}+M_{1}\left(G_{1}\right)\right] D D\left(G_{2}\right)+\left[p_{1}+4 q_{1}+M_{1}\left(G_{1}\right)\right] G u t\left(G_{2}\right) .
\end{aligned}
$$

Similarly, we have

$$
Y_{1}=\operatorname{Gut}\left(G_{2}\right)\left(p_{1}^{2}+4 p_{1} q_{1}+4 q_{1}^{2}\right)+\left(2 p_{1} q_{1}+4 q_{1}^{2}\right) D D\left(G_{2}\right)+4 q_{1}^{2} W\left(G_{2}\right)
$$

and

$$
Y_{2}=M_{2}\left(G_{2}\right) W\left(G_{1}\right)+\left[2 q_{2}+M_{2}\left(G_{2}\right)\right] D D\left(G_{1}\right)+\left[p_{2}+4 q_{2}+M_{2}\left(G_{2}\right)\right] G u t\left(G_{1}\right) .
$$

Then

$$
\begin{aligned}
& \operatorname{Gut}\left(G_{1} \otimes G_{2}\right) \\
\geq & \max \left\{G u t\left(G_{1}\right)\left(p_{2}^{2}+4 p_{2} q_{2}+4 q_{2}^{2}\right)+\left(2 p_{2} q_{2}+4 q_{2}^{2}\right) D D\left(G_{1}\right)+4 q_{2}^{2} W\left(G_{1}\right)\right. \\
& +M_{1}\left(G_{1}\right) W\left(G_{2}\right)+\left[2 q_{1}+M_{1}\left(G_{1}\right)\right] D D\left(G_{2}\right)+\left[p_{1}+4 q_{1}+M_{1}\left(G_{1}\right)\right] G u t\left(G_{2}\right), \\
& G u t\left(G_{2}\right)\left(p_{1}^{2}+4 p_{1} q_{1}+4 q_{1}^{2}\right)+\left(2 p_{1} q_{1}+4 q_{1}^{2}\right) D D\left(G_{2}\right)+4 q_{1}^{2} W\left(G_{2}\right) \\
& \left.+M_{2}\left(G_{2}\right) W\left(G_{1}\right)+\left[2 q_{2}+M_{2}\left(G_{2}\right)\right] D D\left(G_{1}\right)+\left[p_{2}+4 q_{2}+M_{2}\left(G_{2}\right)\right] G u t\left(G_{1}\right)\right\}
\end{aligned}
$$


and

$\operatorname{Gut}\left(G_{1} \otimes G_{2}\right)$

$$
\begin{aligned}
= & \sum_{\{(a, b),(c, d)\} \subseteq V_{1} \times V_{2}} d_{G_{1} \bowtie G_{2}}(a, b) \cdot d_{G_{1} \bowtie G_{2}}(c, d) \cdot d_{G_{1} \bowtie G_{2}}[(a, b),(c, d)] \\
= & \sum_{\{(a, b),(c, d)\} \subseteq V_{1} \times V_{2}}\left[d_{G_{1}}(a)+d_{G_{2}}(b)+d_{G_{1}}(a) d_{G_{2}}(b)\right] \cdot\left[d_{G_{1}}(c)+d_{G_{2}}(d)+d_{G_{1}}(c) d_{G_{2}}(d)\right] \\
& \cdot \max \left\{d_{G_{1}}(a, c), d_{G_{2}}(b, d)\right\} \\
\leq & \sum_{\{(a, b),(c, d)\} \subseteq V_{1} \times V_{2}}\left[d_{G_{1}}(a)+d_{G_{2}}(b)+d_{G_{1}}(a) d_{G_{2}}(b)\right] \cdot\left[d_{G_{1}}(c)+d_{G_{2}}(d)+d_{G_{1}}(c) d_{G_{2}}(d)\right] d_{G_{1}}(a, c) \\
& +\sum_{\{(a, b),(c, d)\} \subseteq V_{1} \times V_{2}}\left[d_{G_{1}}(a)+d_{G_{2}}(b)+d_{G_{1}}(a) d_{G_{2}}(b)\right] \cdot\left[d_{G_{1}}(c)+d_{G_{2}}(d)+d_{G_{1}}(c) d_{G_{2}}(d)\right] d_{G_{2}}(b, d) \\
= & X_{1}+X_{2}+Y_{1}+Y_{2} \\
\leq & G u t\left(G_{1}\right)\left[p_{2}^{2}+4 p_{2} q_{2}+4 q_{2}^{2}+p_{2}+4 q_{2}+M_{2}\left(G_{2}\right)\right]+\left[2 p_{2} q_{2}+4 q_{2}^{2}+2 q_{2}+M_{2}\left(G_{2}\right)\right] D D\left(G_{1}\right) \\
& +\left[4 q_{2}^{2}+M_{2}\left(G_{2}\right)\right] W\left(G_{1}\right)+G u t\left(G_{2}\right)\left[p_{1}^{2}+4 p_{1} q_{1}+4 q_{1}^{2}+p_{1}+4 q_{1}+M_{1}\left(G_{1}\right)\right] \\
& +\left[2 p_{1} q_{1}+4 q_{1}^{2}+2 q_{1}+M_{1}\left(G_{1}\right)\right] D D\left(G_{2}\right)+\left[4 q_{1}^{2}+M_{1}\left(G_{1}\right)\right] W\left(G_{2}\right) .
\end{aligned}
$$

To show the sharpness of the lower bounds of Theorem 3.9, we consider the following example.

Example 1. Let $G$ be a complete graph of order $n$. If $n=2$, then $G=K_{2}$ and $G \otimes G=K_{4}$, and hence $\operatorname{Gut}(G \otimes G)=54=G u t(G)\left[p^{2}+4 p q+4 q^{2}+p+4 q\right]+\left[2 p q+4 q^{2}+2 q+M_{1}(G)\right] D D(G)+\left[4 q^{2}+M_{1}(G)\right] W(G)$. If $n=3$, then $G=K_{3}$ and $G \otimes G=K_{9}$, and hence $\operatorname{Gut}(G \otimes G)=2304=\operatorname{Gut}(G)\left[p^{2}+4 p q+4 q^{2}+p+\right.$ $4 q]+\left[2 p q+4 q^{2}+2 q+M_{1}(G)\right] D D(G)+\left[4 q^{2}+M_{1}(G)\right] W(G)$. From the proof of Theorem 3.9, one can check that $K_{n} \otimes K_{n}$ is an sharp example of the lower bound.

\subsection{For complete product}

We first give the following lemma.

Lemma 3.10. (1) If $A=\left[a_{i j}\right]_{n \times m}$ be any matrix and $I=[1]_{p \times n}$, then $S(I A)=p S(A)$;

(2) If $A=\left[a_{i j}\right]_{m \times n}$ and $I=[1]_{n \times p}$, then $S(A I)=p S(A)$;

(3) If $A=\left[a_{i j}\right]_{p \times m}, I=[1]_{m \times n}$ and $B=\left[b_{i j}\right]_{n \times q}$, then $S(A I B)=S(A) \cdot S(B)$. In particular, if $A=\left[a_{i j}\right]_{n \times n}$ then $S(A I A)=S(A)^{2}$.

Proof. For (1), we have

$$
\begin{aligned}
S(I A) & =\sum_{i=1}^{p} \sum_{j=1}^{m} \sum_{k=1}^{n} 1_{i k} a_{k j}=\sum_{i=1}^{p} \sum_{k=1}^{n} \sum_{j=1}^{m} a_{k j} \\
& =\sum_{i=1}^{p} S(A)=p S(A) .
\end{aligned}
$$


For (2), we have

$$
\begin{aligned}
S(A I) & =\sum_{i=1}^{m} \sum_{j=1}^{p} \sum_{k=1}^{n} a_{i k 1_{k j}}=\sum_{j=1}^{p} \sum_{i=1}^{m} \sum_{k=1}^{n} a_{i k} \\
& =\sum_{j=1}^{p} S(A)=p S(A) .
\end{aligned}
$$

For (3), we have

$$
\begin{aligned}
S(A I B) & =\sum_{i=1}^{p} \sum_{j=1}^{q} \sum_{k=1}^{m} a_{i k} \sum_{s=1}^{n} 1_{k s} b_{s j}=\sum_{i=1}^{p} \sum_{k=1}^{m} a_{i k} \sum_{j=1}^{q} \sum_{s=1}^{n} b_{s j} \\
& =S(A) \cdot S(B) .
\end{aligned}
$$

Corollary 3.11. Let $G$ be $a(p, q)$-graph and $A$ and $K$ be the adjacency matrix of $G$ and $K_{p}$ respectively. Let $I=[1]_{p \times p}$ and $I_{p}$ be the identity matrix. Then $S(A K A)=4 q^{2}-M_{1}(G)$.

Proof. By Lemma 3.10, we have

$$
\begin{aligned}
S(A K A) & =S\left(A\left(I-I_{p}\right) A\right) \\
& =S(A I A)-S\left(A^{2}\right) \\
& =S(A)^{2}-S\left(A^{2}\right)=4 q^{2}-M_{1}(G) .
\end{aligned}
$$

Theorem 3.12. Let $G$ be a $(p, q)$-graph, then

(1) If $\operatorname{diam}(G)=2$, then $G u t(G)=4 q^{2}-M_{1}(G)-M_{2}(G)$.

(2) If $\operatorname{diam}(G)=3$ and $G$ has no cycles of size 3 then

$$
G u t(G)=6 q^{2}-\frac{3}{2} M_{1}(G)-2 M_{2}(G)-N_{2}(G) .
$$

Proof. (1) By Lemma 3.4 and Observation 3.1, we have:

$$
\begin{aligned}
2 G u t(G) & =S\left(A D_{G} A\right)=S(A(A+2 \bar{A}) A)=S\left(A^{3}\right)+2 S(A \bar{A} A) \\
& =2 S(A(A+\bar{A}) A)-S\left(A^{3}\right)=2 S(A K A)-S\left(A^{3}\right) \\
& =8 q^{2}-2 M_{1}(G)-2 M_{2}(G) .
\end{aligned}
$$

(2) By Lemma 3.4 and Observation 3.1, we have:

$$
\begin{aligned}
2 G u t(G) & =S\left(A D_{G} A\right)=S(A(3 K-2 A-B) A) \\
& =3 S(A K A)-2 S\left(A^{3}\right)-S(A B A) \\
& =12 q^{2}-3 M_{1}(G)-4 M_{2}(G)-2 N_{2}(G) .
\end{aligned}
$$


Remark 3.13. Let $A_{1}=\left[a_{i j}\right]_{n_{1} \times n_{1}}$ and $A_{2}=\left[b_{i j}\right]_{n_{2} \times n_{2}}$ be the adjacency matrix of $G_{1}$ and $G_{2}$, respectively. Let $D_{G}$ be distance matrix of graph $G=G_{1} \vee G_{2}$. Let $I_{1}=[1]_{n_{1} \times n_{1}}, I_{2}=[1]_{n_{2} \times n_{2}}, I_{1}^{\prime}=[1]_{n_{1} \times n_{2}}$, $I_{2}^{\prime}=[1]_{n_{2} \times n_{1}}$ and $I_{n}$ be identity matrices. Then

$$
D_{G}=\left(\begin{array}{cc}
2 I_{1}-A_{1}-2 I_{n_{1}}, & I_{1}^{\prime} \\
I_{2}^{\prime}, & 2 I_{2}-A_{2}-2 I_{n_{2}}
\end{array}\right)
$$

is distance matrix of $G_{1} \vee G_{2}$.

Theorem 3.14. Let $G_{1}$ be a graph with order $n_{1}$ and $m_{1}$ edges and $G_{2}$ be a graph with order $n_{2}$ and $m_{2}$ edges. Then

$$
D D\left(G_{1} \vee G_{2}\right)=4\left(n_{1}+n_{2}-1\right)\left(m_{1}+m_{2}+n_{1} n_{2}\right)-M\left(G_{1} \vee G_{2}\right) .
$$

Proof. Since $\operatorname{diam}\left(G_{1} \vee G_{2}\right)=2$, it follows from Lemma 3.3 that

$$
D D\left(G_{1} \vee G_{2}\right)=4\left(n_{1}+n_{2}-1\right)\left(m_{1}+m_{2}+n_{1} n_{2}\right)-M_{1}\left(G_{1} \vee G_{2}\right) .
$$

For computing $M_{1}\left(G_{1} \vee G_{2}\right)$, let $A$ be the adjacency matrix of graph $G=G_{1} \vee G_{2}$. Then

$$
\begin{aligned}
M_{1}\left(G_{1} \vee G_{2}\right) & =S\left(A^{2}\right)=S\left[\left(\begin{array}{cc}
A_{1}, & I_{1}^{\prime} \\
I_{2}^{\prime}, & A_{2}
\end{array}\right)\left(\begin{array}{cc}
A_{1}, & I_{1}^{\prime} \\
I_{2}^{\prime}, & A_{2}
\end{array}\right)\right] \\
& =S\left(\begin{array}{cc}
A_{1}^{2}+I_{1}^{\prime} I_{2}^{\prime}, & A_{1} I_{1}^{\prime}+I_{1}^{\prime} A_{2} \\
I_{2}^{\prime} A_{1}+A_{2} I_{2}^{\prime}, & I_{2}^{\prime} I_{1}^{\prime}+A_{2}^{2}
\end{array}\right) \\
& =S\left(A_{1}^{2}\right)+S\left(I_{1}^{\prime} I_{2}^{\prime}\right)+S\left(A_{1} I_{1}^{\prime}\right)+S\left(I_{1}^{\prime} A_{2}\right) \\
& +S\left(I_{2}^{\prime} A_{1}\right)+S\left(A_{2} I_{2}^{\prime}\right)+S\left(I_{2}^{\prime} I_{1}^{\prime}\right)+S\left(A_{2}^{2}\right) \\
& =M_{1}\left(G_{1}\right)+n_{1}^{2} n_{2}+4 n_{2} m_{1}+4 n_{1} m_{2}+n_{2}^{2} n_{1}+M_{1}\left(G_{2}\right) .
\end{aligned}
$$

Theorem 3.15. Let $G_{1}$ be an $\left(n_{1}, m_{1}\right)$-graph and let $G_{2}$ be an $\left(n_{2}, m_{2}\right)$-graph. Then

$$
\operatorname{Gut}\left(G_{1} \vee G_{2}\right)=4\left(m_{1}+m_{2}+n_{1} n_{2}\right)^{2}-M_{1}\left(G_{1} \vee G_{2}\right)-M_{2}\left(G_{1} \vee G_{2}\right) .
$$

Proof. Let $A_{1}=\left[a_{i j}\right]_{n_{1} \times n_{1}}$ and $A_{2}=\left[b_{i j}\right]_{n_{2} \times n_{2}}$ be the adjacency matrix of $G_{1}$ and of $G_{2}$ respectively. Let $D_{G}$ be distance matrix of graph $G=G_{1} \vee G_{2}$. If we set $I_{1}=[1]_{n_{1} \times n_{1}}, I_{2}=[1]_{n_{2} \times n_{2}}, I_{1}^{\prime}=[1]_{n_{1} \times n_{2}}$, $I_{2}^{\prime}=[1]_{n_{2} \times n_{1}}$ and $I_{n}$ be identity matrix, then it follows from Theorem 3.12 that

$$
\operatorname{Gut}\left(G_{1} \vee G_{2}\right)=4\left(m_{1}+m_{2}+n_{1} n_{2}\right)^{2}-M_{1}\left(G_{1} \vee G_{2}\right)-M_{2}\left(G_{1} \vee G_{2}\right),
$$

since $\operatorname{diam}\left(G_{1} \vee G_{2}\right)=2$.

For computing $M_{2}\left(G_{1} \vee G_{2}\right)$, let $A$ be the adjacency matrix of graph $G=G_{1} \vee G_{2}$. Then 


$$
\begin{aligned}
& 2 M_{2}\left(G_{1} \vee G_{2}\right) \\
& =S\left(A^{3}\right)=S\left[\left(\begin{array}{cc}
A_{1}, & I_{1}^{\prime} \\
I_{2}^{\prime}, & A_{2}
\end{array}\right)\left(\begin{array}{cc}
A_{1}, & I_{1}^{\prime} \\
I_{2}^{\prime}, & A_{2}
\end{array}\right)\left(\begin{array}{cc}
A_{1}, & I_{1}^{\prime} \\
I_{2}^{\prime}, & A_{2}
\end{array}\right)\right] \\
& =S\left[\left(\begin{array}{cc}
A_{1}^{2}+I_{1}^{\prime} I_{2}^{\prime} & A_{1} I_{1}^{\prime}+I_{1}^{\prime} A_{2} \\
I_{2}^{\prime} A_{1}+A_{2} I_{2}^{\prime} & I_{2}^{\prime} I_{1}^{\prime}+A_{2}^{2}
\end{array}\right)\left(\begin{array}{cc}
A_{1}, & I_{1}^{\prime} \\
I_{2}^{\prime}, & A_{2}
\end{array}\right)\right] \\
& =S\left[\left(\begin{array}{cc}
A_{1}{ }^{3}+I_{1}^{\prime} I_{2}^{\prime} A_{1}+A_{1} I_{1}^{\prime} I_{2}^{\prime}+I_{1}^{\prime} A_{2} I_{2}^{\prime} & A_{1}{ }^{2} I_{1}^{\prime}+I_{1}^{\prime} I_{2}^{\prime} I_{1}^{\prime}+A_{1} I_{1}^{\prime} A_{2}+I_{1}^{\prime} A_{2}{ }^{2} \\
I_{2}^{\prime} A_{1}{ }^{2}+A_{2} I_{2}^{\prime} A_{1}+I_{2}^{\prime} I_{1}^{\prime} I_{2}^{\prime}+A_{2}{ }^{2} I_{2}^{\prime} & I_{2}^{\prime} A_{1} I_{1}^{\prime}+A_{2} I_{2}^{\prime} I_{1}^{\prime}+I_{2}^{\prime} I_{1}^{\prime} A_{2}+A_{2}{ }^{3}
\end{array}\right)\right] \\
& =S\left(A_{1}^{3}\right)+S\left(I_{1}^{\prime} I_{2}^{\prime} A_{1}\right)+S\left(A_{1} I_{1}^{\prime} I_{2}^{\prime}\right)+S\left(I_{1}^{\prime} A_{2} I_{2}^{\prime}\right) \\
& +S\left(A_{1}^{2} I_{1}^{\prime}\right)+S\left(I_{1}^{\prime} I_{2}^{\prime} I_{1}^{\prime}\right)+S\left(A_{1} I_{1}^{\prime} A_{2}\right)+S\left(I_{1}^{\prime} A_{2}^{2}\right) \\
& +S\left(I_{2}^{\prime} A_{1}{ }^{2}\right)+S\left(A_{2} I_{2}^{\prime} A_{1}\right)+S\left(I_{2}^{\prime} I_{1}^{\prime} I_{2}^{\prime}\right)+S\left(A_{2}{ }^{2} I_{2}^{\prime}\right) \\
& +S\left(I_{2}^{\prime} A_{1} I_{1}^{\prime}\right)+S\left(A_{2} I_{2}^{\prime} I_{1}^{\prime}\right)+S\left(I_{2}^{\prime} I_{1}^{\prime} A_{2}\right)+S\left(A_{2}{ }^{3}\right) .
\end{aligned}
$$

From Lemma 3.10, we have

$$
\begin{aligned}
2 M_{2}\left(G_{1} \vee G_{2}\right) & =2 M_{2}\left(G_{1}\right)+4 n_{1} n_{2} m_{1}+2 n_{2} M_{1}\left(G_{1}\right)+2 n_{1}^{2} n_{2}^{2}+8 m_{1} m_{2} \\
& +2 n_{1}^{2} m_{2}+2 n_{1} M_{1}\left(G_{2}\right)+2 n_{2}^{2} m_{1}+4 n_{1} n_{2} m_{2}+2 M_{2}\left(G_{2}\right),
\end{aligned}
$$

and hence

$$
\begin{aligned}
M_{2}\left(G_{1} \vee G_{2}\right) & =M_{2}\left(G_{1}\right)+M_{2}\left(G_{2}\right)+n_{2} M_{1}\left(G_{1}\right)+n_{1} M_{1}\left(G_{2}\right) \\
& +2 n_{1} n_{2} m_{2}+2 n_{1} n_{2} m_{1}+n_{1}^{2} n_{2}^{2}+4 m_{1} m_{2}+n_{1}^{2} m_{2}+n_{2}^{2} m_{1} \\
& =M_{2}\left(G_{1}\right)+M_{2}\left(G_{2}\right)+n_{2} M_{1}\left(G_{1}\right)+n_{1} M_{1}\left(G_{2}\right) \\
& +\left(n_{1} n_{2}+2 m_{2}\right)\left(n_{1} n_{2}+2 m_{1}\right)+n_{1}^{2} m_{2}+n_{2}^{2} m_{1} .
\end{aligned}
$$

\section{References}

[1] A. Alwardi, B. Arsić, I. Gutman, N. D. Soner, The common neighborhood graph and its energy, Iran. J. Math. Sci. Inf. 7 (2012) 1-8.

[2] J. A. Bondy, U. S. R. Murty, Graph theory, Springer, New York, 2008.

[3] A. S. Bonifácio, R. R. Rosa, I. Gutman, N. M. M. de Abreu, Complete common neighborhood graphs, Proceedings of Congreso Latino-Iberoamericano de Investigaci on Operativa and Simposio Brasileiro de Pesquisa Operacional (2012) 4026-4032.

[4] S. Chen, Cacti with the smallest, second smallest, and third smallest Gutman index, J. Combin. Optim. 31(1) (2016) 327-332. 
[5] S. Chen, Z. Guo, A lower bound on the degree distance in a tree, Int. J. Contemp. Math. Sci. 5(13) (2010) 649-652.

[6] P. Dankelmann, I. Gutman, S. Mukwembi, H.C. Swart, On the degree distance of a graph, Discrete Appl. Math. 157(13) (2009) 2773-2777.

[7] P. Dankelmann, I. Gutman, S. Mukwembi, H. C. Swart, The edge-Wiener index of a graph, Discrete Math. 309 (2009) 3452-457.

[8] A. A. Dobrynin, R. Entringer, I. Gutman, Wiener index of trees: Theory and applications, Acta Appl. Math. 66 (2001) 211-249.

[9] A. A. Dobrynin, A. A. Kochetova, Degree distance of a graph: A degree analogue of the Wiener index, J. Chem. Inf. Comput. Sci. 34(5) (1994) 1082-1086.

[10] T. Došlić, B. Furtula, A. Graovac, I. Gutman, S. Moradi, Z. Yarahmadi, On vertex-degree-based molecular structure descriptors, MATCH Commun. Math. Comput. Chem. 66 (2011) 613-626.

[11] A. A. Dobrynin, I. Gutman, S. Klavžar, P. Žigert, Wiener index of hexagonal systems, Acta Appl. Math. 72 (2002) 247-294.

[12] L. Feng, W. Liu, The maximal Gutman index of bicyclic graphs, MATCH Commun. Math. Comput. Chem. 66 (2011) 699-708.

[13] I. Gutman, Y. N. Yeh, S. L. Lee, Y. L. Luo, Some recent results in the theory of the Wiener number, Indian J. Chem. 32A (1993) 651-661.

[14] I. Gutman, Selected properties of the Schultz molecular topological index, J. Chem. Inf. Comput. Sci. 34(5) (1994) 1087-1089.

[15] I. Gutman, N. Trinajstić, Graph theory and molecular orbitals. Total $\pi$-electron energy of alternant hydrocarbons, Chem. Phys. Lett. 17(4) (1972) 535-538.

[16] P. Paulraja, V.S. Agnes, Degree distance of product graphs, Discrete Math., Alg. and Appl. 6(1) (2014) 1450003.

[17] P. Paulraja, V. S. Agnes, Gutman index of product graphs, Discrete Math., Alg. and Appl. 6(4) (2014) 1450058.

[18] R. Hammack, W. Imrich, Sandi Klavžr, Handbook of product graphs, Second edition, CRC Press, 2011.

[19] S. Nikolić, N. Trinajstić, Z. Mihalić, The Wiener index: Development and applications, Croat. Chem. Acta 68 (1995) 105-129.

[20] H. Wiener, Structural determination of paraffin boiling points, J. Am. Chem. Soc. 69 (1947) 17-20.

[21] H.P. Schultz, Topological organic chemistry. 1. Graph theory and topological indices of alkanes, J. Chem. Inf. Comput. Sci. 29(3) (1989) 227-228. 